\title{
Parsing with the Shortest Derivation
}

\author{
Rens Bod \\ Informatics Rescarch Institute, University of Leeds, Leeds LS2 9JT, \& \\ Institute for Logic, Language and Computation, University of Amsterdam \\ rens@scs.leeds.ac.uk
}

\begin{abstract}
Common wisdom has it that the bias of stochastic grammars in favor of shorter derivations of a sentence is harmful and should be redressed. We show that the common wisdom is wrong for stochastic grammars that use elementary trees instead of context-frec rulcs, such as Stochastic Tree-Substitution Grammars used by Data-Oriented Parsing models. For such grammars a non-probabilistic metric based on the shortest derivation outperforms a probabilistic metric on the $\Lambda$ TIS and OVIS corpora, while it obtains competitive results on the Wall Street Journal (WSJ) corpus. This paper also contains the first published experiments with DOP on the WSJ.
\end{abstract}

\section{Introduction}

A well-known property of stochastic grammars is their propensity to assign higher probabilitics to shorter derivations of a sentence (cl. Chitrao \& Grishman 1990; Magerman \& Marcus 1991; Briscoe \& Carroll 1993; Charniak 1996). This propensity is due to the probability of a derivation being computed as the product of the rule probabilities, and thus shorter derivations involving fewer rules tend to have higher probabilities, almost regardless of the training data. While this bias may seem intercsting in the light of the principle of cognitive economy, shorter derivations generate smaller parse trees (consisting of fewer nodes) which ate not warranted by the correct parses of sentences. Most systems therefore redress this bias, for instance by normalizing the derivation probability (see Caraballo \& Charniak 1998).

However, for stochastic grammars that use elementary trees instead of context-free rules, the propensity to assign higher probabilities to shorter derivations does not necessarily lead to a bias in favor of smaller parse trees, because elementary trees may differ in size and lexicalization. For Stochastic Tree-Substitution Grammars (STSG) used by DataOriented Parsing (DOP) models, it has been observed that the shortest derivation of a sentence consists of the largest subtrees seen in a treebank that gencrate that sentence (ef. Bod 1992, 98). We may therefore wonder whether for STSG the bias in favor of shonter derivations is perhaps beneficial rather than harmful.

To investigate this question we created a new STSG-DOP model which uses this bias as a feature. This non-probabilistic DOP model parses each sentence by relurning its shortest derivation (consisting of the fewest subtrees seen in the corpus). Only if there is more than one shortest derivation the model backs ofl to a frequency ordering of the corpussubtrees and chooses the shortest derivation with most highest ranked subtrees. We compared this nonprobabilistic DOP model against the probabilistic DOP model (which estimates the most probable parse for each sentence) on three different domains: the Penn A'TIS trecbank (Marcus et al. 1993), the Dutch OVIS treebank (Bonnemat et al. 1997) and the Penn Wall Street Journal (WSJ) treebank (Marcus et al. 1993). Surprisingly, the non-probabilistic DOP model outperforms the probabilistic DOP model on both the ATIS and OVIS trecbanks, while it obtains competitive results on the WSJ treebank. We conjecture that any stochastic grammar which uses units of flexible size can be turned into an accurate non-probabilistic version.

The rest of this paper is organized as follows: we first explain both the probabilistic and nonprobabilistic DOP model. Next, we go into the computational aspects of these models, and finally we compare the performance of the models on the three trecbanks.

\section{Probabilistic vs. Non-Probabilistic Data-Oriented Parsing}

Both probabilistic and non-probabilistic DOP are based on the DOP model in Bod (1992) which extracts a Stochastic Trec-Substitution Grammar from a trecbank ("STSG-DOP").' STSG-DOP uses subtrecs

\footnotetext{
${ }^{1}$ Note that the DOP-approach of extracting grammars from corpora has been applied to a wide variely of other grammatical frameworks, including Tree-Inscrtion Grammar
} 
from parse trees in a corpus as elementary trees, and leftmost-substitution to combine subtrees into new trees. As an example, consider a very simple corpus consisting of only two trees (we leave out some subcategorizations to keep the example simple):
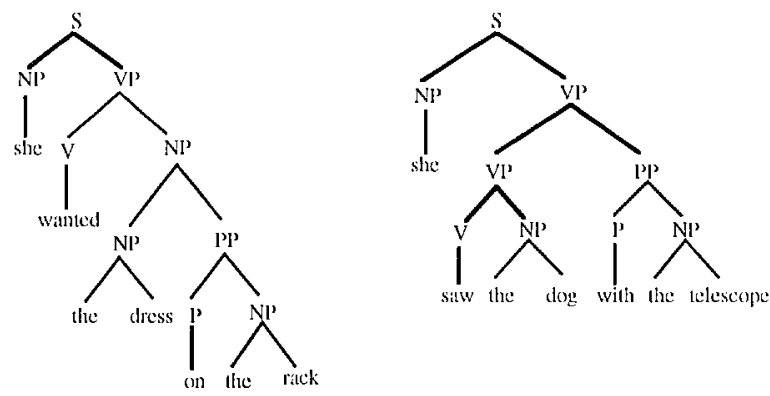

Figure 1. A simple corpus of two trees.

A new sentence such as She saw the dress with the telescope can be parsed by combining subtrees from this corpus by means of leftmost-substitution (indicated as ${ }^{\circ}$ ):
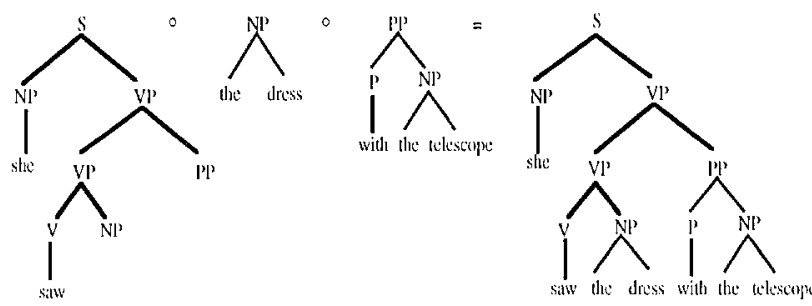

Figure 2. Derivation and parse tree for the sentence She sav the dress with the telescope

Note that other derivations, involving different subtrees, may yield the same parse tree; for instance:
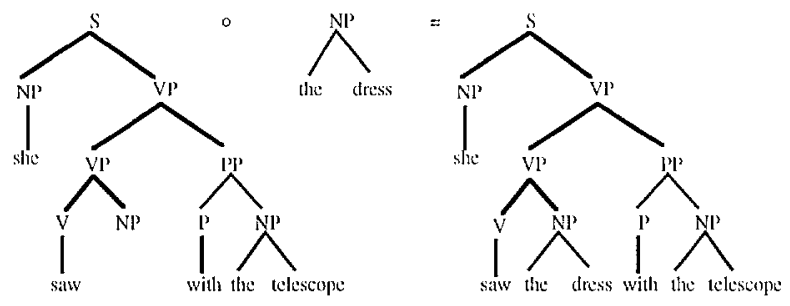

Figure 3. Different derivation yielding the same parse tree for She saw the dress with the telescope

Note also that, given this example corpus, the sentence we considered is ambiguous; by combining

(Hoogweg 2000), Trec-Adjoining Grammar (Neumann 1998), Lexical-Functional Grammar (Bod \& Kaplan 1998; Way 1999; Bod 2000a), Head-driven Phrase Structure Grammar (Neumann \& Flickinger 1999), and Montaguc Grammar (van den Berg et al. 1994; Bod 1998). For the relation between DOP and Memory-Based Learning, see Daclemans (1999). other subtrees, a different parse may be derived, which is analogous to the first rather than the second corpus sentence:
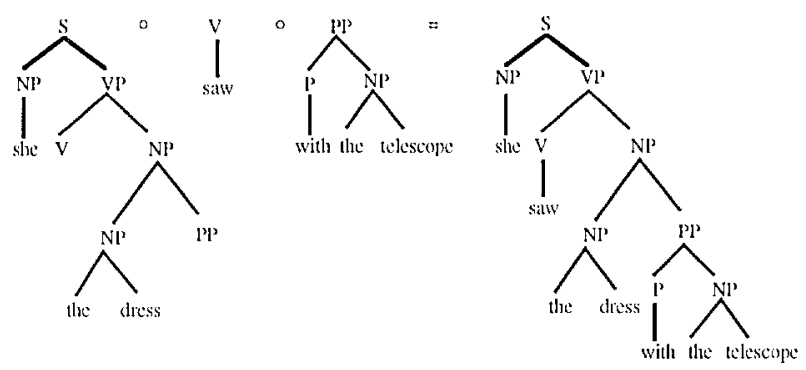

Figure 4. Different derivation yiclding a different parse tree for She saw the dress with the telescope

The probabilistic and non-probabilistic DOP models differ in the way they define the best parse tree of a sentence. We now discuss these models separately.

\subsection{The probabilistic DOP model}

The probabilistic DOP model introduced in Bod $(1992,93)$ computes the most probable parse tree of a sentence from the normalized subtrec frequencies in the corpus. The probability of a subtree $t$ is estimated as the number of occurrences of $t$ seen in the corpus, divided by the total number of occurrences of corpussubtrecs that have the same root label as $t$. Let $|t|$ return the number of occurrences of $t$ in the corpus and let $r(t)$ return the root label of $t$ then: $P(t)=|t| / \sum_{t^{\prime}: r\left(t^{\prime}\right)=r(t)}\left|t^{\prime}\right| .^{2}$ The probability of a derivation is computed as the product of the probabilities of the subtrees involved in it. The probability of a parse tree is computed as the sum of the probabilities of all distinct derivations that produce that tree. The parse tree with the highest

\footnotetext{
2 It should be stressed that there may be several other ways to estimate subtree probabilitics in DOP. For example, Bonnema et al. (1999) estimate the probability of a subtree as the probability that it has been involved in the derivation of a corpus tree. It is not yet known whether this alternative probability model outperforms the model in Bod (1993). Johnson (1998) pointed out that the subtree estimator in Bod (1993) yields a statistically inconsistent model. This means that as the training corpus increases the corresponding sequences of probability distributions do not converge to the true distribution that generated the training data. Experiments with a consistent maximum likelihood estimator (based on the inside-outside algorithm in Lari and Young 1990), leads however to a significant decrease in parse accuracy on the ATIS and OVIS corpora. This indicates that statistically consistency does not necessarily lead to better performance.
} 
probability is defined as the best parse tree of a sentence.

The probabilistic DOP model thus considers counts of subtres of a wide range of sizes in computing the probability of a tree: everything from counts of single-level rules to counts of entire trees.

\subsection{The non-probabilistic DOP model}

The non-probabilistic DOP model uses a rather different definition of the best parse tree. Insteat of computing the most probable parse of a sentence, it computes the parse tree which can be generated by the fewest corpus-subtrees, i.c., by the shortest derivation independent of the subtree probabilities. Since subtrees are allowed to be of arbitrary size, the shortest derivation typically corresponds to the parse tree which consists of largest possible corpussubtrees, thus maxinizing syntactic context. lior example, given the corpus in Figure 1, the best parse tree for She saw the dress with the telescope is given in Figure 3, since that parse tree can be generated by a derivation of only two corpus-subtrees, while the parse tree in ligure 4 needs at least three corpussubtrees to be generated. (Interestingly, the parse tree with the shortest derivation in Figure 3 is also the most probable parse tree according 10 probabilistic DOP for this corpus, but this need not always be so. As mentioned, the probabilistic DOP model has already a bias to assign higher probabilities to parse trees that can be generated by shorter derivations. The non-probabilistic DOP model makes this bias absolute.)

The shortest derivation may not be unique: it may happen that different parses of a sentence are generated by the same minimal number of corpussubtrees. In that case the model backs off to a frequency ordering of the subtrees. That is, all subtrees of each root label are assigned a rank according to their frequency in the corpus: the most frequent subtrec (or subtrees) of each root label get rank 1, the second most frequent subtrec gets rank 2 , etc. Next, the rank of each (shortest) derivation is computed as the sum of the ranks of the subtrees involved. The derivation witl the smallest sum, or highest rank, is taken as the best derivation producing the best parse tree.

The way we compute the rank of a derivation by summing up the ranks of its subtrees may secm rather ad hoc. However, it is possible to provide an information-theoretical motivation for this model. According to Zipl's law, rank is roughly proportional to the negative logarithm of frequency (Zipl 1935). In Shannon's Information Theory (Shannon 1948), the negative logarithm (of base 2) of the probability of an event is better known as the information of that event. Thus, the rank of a subtree is roughly proportional to its information. It follows that minimizing the sum of the subtree ranks in a derivation corresponds to minimizing the (self-)information of a derivation.

\section{Computational Aspects}

\subsection{Computing the most probable parse}

Bod (1993) showed how standard chart parsing technigues can be applicd to probabilistic DOP. Each corpus-subtree $t$ is converted into a context-free rule $r$ where the lefthand side of $r$ corresponds to the root label of $t$ and the righthand side of $r$ corresponds to the frontier labels of $t$. Indices link the mes to the original subtrees so ats to maintain the subtrec's internal structure and probability. These rules are used to create a derivation forest for a sentence, and the most probable parse is computed by sampling a sufficiently large number of random derivations from the forest ("Monte Carlo disambiguation", see Bod 1998; Chappelier \& Rajman 2000). While this technique has been successfully applied to parsing the ATIS portion in the Penn Treebank (Marcus et al. 1993), it is extremely time consuming. This is mainly becatse the number of random derivations that should be sampled to reliably estimate the most probable parse increases exponentially with the sentence length (see Goodman 1998). It is therefore questionable whether Bod's sampling technique can be scaled to larger corpora such as the OVIS and the WSJ corpora.

Goodman (1998) showed how the probabilistic DOP model can be reduced to a compact stochastic context-free grammar (SCFG) which contains exactly eight SCFG rules for cach node in the training set trees. Although Goodman's reduction method does still not allow for an efficient computation of the most probable parse in DOP (in fact, the problem of computing the most probable parse is NP-hard - see Sima'an 1996), his method does allow for an efficient computation of the "maximum constituents parse", i.c., the parse tree that is most likely to have the largest number of correct constiuents (also called the "labeled recall parse"). Goodman has shown on the ATIS corpus that the maximum constituents parse performs at least as well as the most probable parse if all subtrees are used. Unfortunately, Goodman's reduction method remains 
beneficial only if indeed all trecbank subtrees are used (sec Sima'an 1999: 108), while maximum parse accuracy is typically obtained with a subtrec set which is smaller than the total set of subtrees (this is probably due to data-sparseness effects -- sec Bonnema et al. 1997; Bod 1998; Sima'an 1999).

In this paper we will use Bod's subtree-to-rule conversion method for studying the bchavior of probabilistic against non-probabilistic DOP for different maximum subtree sizes. However, we will not use Bod's Monte Carlo sampling technique from complete derivation forests, as this turns out to be computationally impractical for our larger corpora. Instcad, we use a Viterbi $n$-best search and estimate the most probable parse from the 1,000 most probable derivations, summing up the probabilities of derivations that generate the same tree. The algorithm for computing $n$ most probable derivations follows straightforwardly from the algorithm which computes the most probable derivation by means of Viterbi optimization (sec Sima'an 1995, 1999).

\subsection{Computing the shortest derivation}

As with the probabilistic DOP model, we first convert the corpus-subtrees into rewrite rules. Next, the shortest derivation can be computed in the same way as the most probable derivation (by Viterbi) if we give all rules equal probabilities, in which case the shortest derivation is equal to the most probable derivation. This can be seen as follows: if each rule has a probability $p$ then the probability of a derivation involving $n$ rules is equal to $p^{n}$, and since $0<p<1$ the derivation with the fewest rules has the greatest probability. In our experiments, we gave each rule a probability mass equal to $1 / R$, where $R$ is the number of distinct rules derived by Bod's method.

As mentioned above, the shortest derivation may not be unique. In that case we compute all shortest derivations of a sentence and then apply our ranking scheme to these derivations. Note that this ranking scheme does distinguish between subtrees or different root labels, as it ranks the subtrees given their root label. The ranks of the shortest derivations are computed by summing up the ranks of the subtrees they involve. The shortest derivation with the smallest sum of subtree ranks is taken to produce the best parse tree. ${ }^{3}$

\footnotetext{
${ }^{3}$ It may happen that different shortest derivations generate the same tree. We will not distinguish between these cases, however, and compute only the shortest derivation with the highest rank.
}

\section{Experimental Comparison}

\subsection{Experiments on the ATIS corpus}

For our first comparison, we used 10 splits from the Penn ATIS corpus (Marcus et al. 1993) into training sets of 675 sentences and test sets of 75 sentences. These splits were random except for one constraint: that all words in the test set actually occurred in the training set. As in Bod (1998), we climinated all epsilon productions and all "pseudo-attachments". As accuracy metric we used the exact match defined as the percentage of the best parse trees that are identical to the test set parses. Since the Penn ATIS portion is relatively small, we were able to compute the most probable parse both by means of Monte Carlo sampling and by means of Viterbi $n$-best. Table 1 shows the means of the exact match accuracies for increasing maximum subtrce depths (up to depth 6).

\begin{tabular}{cccc}
\hline $\begin{array}{c}\text { Depth of } \\
\text { subtrces }\end{array}$ & \multicolumn{2}{c}{ Probabilistic DOP } & Non-probabilistic \\
Monte Carlo & Viterbi $n$-best & DOP \\
\hline 1 & 46.7 & 46.7 & 24.8 \\
$\leq 2$ & 67.5 & 67.5 & 40.3 \\
$\leq 3$ & 78.1 & 78.2 & 57.1 \\
$\leq 4$ & 83.6 & 83.0 & 81.5 \\
$\leq 5$ & 83.9 & 83.4 & 83.6 \\
$\leq 6$ & 84.1 & 84.0 & 85.6 \\
\hline
\end{tabular}

Table 1. Exact match accuracies for the ATIS corpus

The table shows that the two methods for probabilistic DOP score roughly the same: at depth $\leq 6$, the Monte Carlo method obtains $84.1 \%$ while the Viterbi $n$-best method obtains $84.0 \%$. These differences are not statistically significant. The table also shows that for small subtree depths the non-probabilistic DOP model performs considerably worse than the probabilistic model. This may not be surprising since for small subtrees the shortest derivation corresponds to the smallest parse trec which is known to be a bad prediction of the correct parse tree. Only if the subtrees are larger than depth 4, the non-probabilistic DOP model scores roughly the same as its probabilistic counterpart. At subtrec depth $\leq 6$, the non-probabilistic DOP model scores $1.5 \%$ better than the best score of the probabilistic DOP model, which is statistically significant according to paired $t$-tests.

\subsection{Experiments on the OVIS corpus}

For our comparison on the OVIS corpus (Bonnema et al. 1997; Bod 1998) we again used 10 random splits under the condition that all words in the test set occurred in the training set $(9000$ sentences for 
training, 1000 sentences for testing). The OVIS trees contain both syntactic and semantic annotations, but no epsilon productions. $\Lambda s$ in Bod (1998), we treated the syntactic and scmantic annotations of each node as one label. Consequently, the labels are very restrictive and collecting statistics over them is difficult. Bonnema et al. (1997) and Sima'an (1999) report that (probabilistic) DOP suffers considerably from data-sparseness on OVIS, yielding a decrease in parse accuracy if subtrees larger than depth 4 are included. Thus it is interesting to investigate how nonprobabilistic DOP behaves on this corpus. Table 2 shows the means of the exact match accuracies for increasing subtree depths.

\begin{tabular}{ccc}
\hline $\begin{array}{l}\text { Depth of } \\
\text { Subtres }\end{array}$ & $\begin{array}{c}\text { Probabilistic } \\
\text { J)OP' }\end{array}$ & $\begin{array}{c}\text { Non-probabilistic } \\
\text { DoP' }\end{array}$ \\
\hline 1 & 83.1 & 70.4 \\
$\leq 2$ & 87.6 & 85.1 \\
$\leq 3$ & 89.6 & 89.5 \\
$\leq 4$ & 90.0 & 90.9 \\
$\leq 5$ & 89.7 & 91.5 \\
$\leq 6$ & 88.8 & 92.2 \\
\hline
\end{tabular}

Table 2. Exact match accuracies for the OVIS corpus

We again see that the non-probabilistic DoP model performs badly for small subtree depths while it outperforms the probabilistic DOP model if the subtrees get larger (in this case for depth $>3$ ). But while the accuracy of probabilistic DOP deterionates after depth 4, the accuracy of non-probabilistic IOOP continues to grow. Thus non-probabilistic J)OP secms relatively insensitive to the low frequency of larger subtrees. This property may be especially uselul il no meaninglul statistics can be collected while sentences can still be parsed by large chunks. $\Lambda \mathrm{t}$ depth $\leq 6$, non-probabilistic DOP scores $3.4 \%$ better than probabilistic DOP, which is statistically signilicant using paired $t$-tests.

\subsection{Experiments on the WSJ corpus}

Both the ATIS and OVIS corpus represent restricted domains. In order to extend our results to a broadcoverage domain, we tested the two models also on the Wall Street Jounal portion in the Penn Treebank (Marcus et al. 1993).

To make our results comparable to others, we did not test on different random splits but used the now standard division of the WSJ with sections 2-21 for training (approx. 40,000 sentences) and section 23 for testing (sec Collins 1997, 1999; Charniak 1997 ,
2000); Ratnaparkhi 1999); we only tested on sentences $\leq 40$ words (2245 sentences). All trees were stripped ofl their semantic lags, co-reference information and quotation marks. We used all training set subtrees of depth 1 , but due to memory limitations we used a subset of the subtrees larger than depth 1 by taking for each depth a random sample of 400,000 subtrecs. No subtrees larger than depth 14 were used. This resulted into a total set of $5,217,529$ subtrees which were smoothed by Good-Turing (see Bod 1996). We did not employ a separate part-of-speech tagger: the test sentences were directly parsed by the training set subtrees. For words that were unknown in the training set, we guessed their categories by means of the method described in Weischedel et al. (1993) which uses statistics on word-endings, hyphenation and capialization. The guessed category for cach unknown word was converted into a depth-1 subtrec and assigned a probability (or frequency for nonprobabilistic 1)OP') by means of simple Good-Turing.

$\Lambda$ s accuracy metric we used the standard PARSLVAL, scores (Black et al. 1991) to compare a proposed parse $P$ with the corresponding correct trecbank parse 7 as follows:

$$
\begin{aligned}
& \text { Labeled precision }=\frac{\# \text { correct constituents in } P}{\# \text { constituents in } P} \\
& \text { Labeled Recall }=\frac{\# \text { correct constituents in } P}{\# \text { constitucnts in } T}
\end{aligned}
$$

$A$ constituent in $P$ is "correct" il there exists a constituent in $T$ of the same label that spans the same words. As in other work, we collapsed ADVP and PRT to the same label when calculating these scores (see Collins 1997; Ratnaparkhi 1999; Channiak 1997).

Table 3 shows the labeled precision (LP) and labeled recall (LR) scores for probabilistic and nonprobabilistic DOP for six different maximum subtrce depths.

\begin{tabular}{ccccc}
\hline $\begin{array}{l}\text { Depthol } \\
\text { subtrees }\end{array}$ & \multicolumn{2}{c}{ Probabilistic DOP } & \multicolumn{2}{c}{$\begin{array}{c}\text { Non-probabilistic } \\
\text { DOP }\end{array}$} \\
& LP & L.R & LP' $^{\text {LP }}$ & LR \\
\hline$\leq 4$ & 84.7 & 84.1 & 81.6 & 80.1 \\
$\leq 6$ & 86.2 & 86.0 & 85.0 & 84.7 \\
$\leq 8$ & 87.9 & 87.1 & 87.2 & 87.0 \\
$\leq 10$ & 88.6 & 88.0 & 86.8 & 86.5 \\
$\leq 12$ & 89.1 & 88.8 & 87.1 & 86.9 \\
$\leq 14$ & 89.5 & 89.3 & 87.2 & 86.9 \\
\hline
\end{tabular}

Table 3. Scores on the WSJ corpus (sentences $\leq 40$ words) 
The table shows that probabilistic DOP outperforms non-probabilistic DOP for maximum subtrec depths 4 and 6 , while the models yicld rather similar results for maximum subtree depth 8. Surprisingly, the scores of non-probabilistic DOP deteriorate if the subtrees are further enlarged, while the scores of probabilistic DOP continue to grow, up to $89.5 \% \mathrm{LP}$ and $89.3 \%$ L.R. These scores are higher than those of several other parsers (e.g. Collins 1997, 99; Charniak 1997), but remain behind the scores of Charniak (2000) who obtains $90.1 \%$ LP and $90.1 \%$ LR for sentences $\leq 40$ words. However, in Bod (2000b) we show that even higher scores can be obtained with probabilistic DOP by restricting the number of words in the subtrec frontiers to 12 and restricting the depth of unlexicalized subtrees to 6 ; with these restrictions an $\mathrm{LP}$ of $90.8 \%$ and an LR of $90.6 \%$ is achieved.

We may raise the question as to whether we actually need these extremely large subtrees to obtain our best results. One could argue that DOP's gain in parse accuracy with increasing subtree depth is due to the model becoming sensitive to the influence of lexical heads higher in the tree, and that this gain could also be achieved by a more compact depth-1 DOP model (i.e. an SCFG) which annotates the nonterminals with headwords. However, such a headlexicalized stochastic grammar does not capture dependencies between nonheadwords (such as more and than in the WSJ construction carry more people than cargo where neither more nor than are headwords of the NP-constituent more people than cargo), whereas a frontier-lexicalized DOP model using large subtrees does capture these dependencies since it includes subtrees in which e.g. more and than are the only frontier words. In order to isolate the contribution of nonheadword dependencies, we eliminated all subtrees containing two or more nonheadwords (where a nonheadword of a subtree is a word which is not a headword of the subtrec's root nonterminal -- although such a nonheadword may be a headword of one of the subtree's internal nodes). On the WSJ this led to a decrease in LP/LR of $1.2 \% / 1.0 \%$ for probabilistic DOP. Thus nonheadword dependencies contribute to higher parse accuracy, and should not be discarded. This goes against common wisdom that the relevant lexical dependencies can be restricted to the locality of headwords of constituents (as advocated in Collins 1999). It also shows that DOP's frontier lexicalization is a viable alternative to constituent lexicalization (as proposed in Charniak 1997; Collins 1997, 99; Eisner 1997). Moreover, DOP's use of large subtrees makes the model not only more lexically but also more structurally sensitive.

\section{Conclusion}

Common wisdom has it that the bias of stochastic grammars in favor of shorter derivations is harmful and should be redressed. We have shown that the common wisdom is wrong for stochastic treesubstitution grammars that use elementary trees of llexible size. For such grammars, a non-probabilistic metric based on the shortest derivation outperlorms a probabilistic metric on the ATIS and OVIS corpora, while it obtains competitive results on the Wall Street Journal corpus. We have seen that a nonprobabilistic version of DOP performed especially well on corpora for which collecting subtrec statistics is difficult, while sentences can still be parsed by relatively large chunks. We have also seen that probabilistic DOP obtains very competitive results on the WSJ corpus. Finally, we conjecture that any stochastic grammar which uses elementary trees rather than context-free rules can be turned into an accurate non-probabilistic version (e.g. Tree-Insertion Grammar and Tree-Adjoining Grammar).

\section{Acknowledgements}

Thanks to Khalil Sima'an and threc anonymous reviewers for useful suggestions.

\section{References}

Berg, M. van den, R. Bod and R. Scha, 1994. "A CorpusBased Approach to Semantic Interpretation", Proceedings Ninth Amsterdam Colloquium, Amsterdam, The Netherlands.

Black, E. et al. 1991., A Procedure for Quantitatively Comparing the Syntactic Coverage of English, Proceedings DARPA Speech and Natural Language Workshop, Pacilic Grove, Morgan Kaufmann.

Bod, R. 1992. "Data-Oriented Parsing (DOP)", Proceedings COLING-92, Nantes, France.

Bod, R. 1993. "Using an Annotated Corpus as a Stochastic Grammar", Proceedings European Chapter of the ACL'93, Utrecht, The Netherlands.

Bod, R. 1996. "T'wo Questions about Data Oriented Parsing", Proceedings Fourth Workshop on Very Large Corpora, Copenhagen, Denmark.

Bod, R. 1998. Beyond Grammar: An Experience-Based Theory of Language, CSLI Publications, Cambridge University Press.

Bod, R. 2000a. "An Empirical Evaluation of LFG-DOP", Proceedings COLING-2000, Saarbrücken, Germany. 
Bod, R. 2000b. "Redundancy and Minimality in Statistical Parsing with the DOP Model", submitted for publication.

Bod, R. and R. Kaplan, 1998. "A Probabilistic CorpusDriven Model for Icxical lunctional Analysis", Proceedings coldNG-ACL'OS, Montreal, Canada.

Bonnema, R., R. Bod and R. Scha, 1997. "A I)(OP' Model for" Semantic Interpretation", Procedings ACH LACL-97. Madricl, Spain.

Bonnema, R., P. Buying and R. Scha, 1999. "A New Probability Model for Data-(Oriented Parsing", Proceedings of the Amsterdam Colloquimm 1909, Ansterdam, The Netherlands.

Briseoe, T. and J. Carroll, 1993. "Generalized Probabilistic l.R Parsing of Natural language (Corpora) with Unification-Based Grammars", Computational Linguistics 19(1), 25-59).

Caraballo, S. and li. Charniak, 1998. "New liggures of Merit for Bost-first Probabilistic chat parsing", Computatonal Lingutistics 24, 275-298.

Chappelier, J. and M. Rajman, 2000. "Monte Carlo

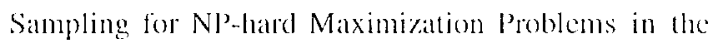
Framework of Weighted Parsing", in Natural Language Processing -- NIIP 2000, Lecture Notes in Artificial Intelligence 1835, D). Christodoulakis (ed.). $2000,106-117$.

Charniak, 1:. 1996. "Tree-bank Grammats", Procecedings AMA-96, Menlo Park, Cal.

Charniak, E. 1997. "Statistical Parsing with a Context-litec Grammar and Word Statistics", Proceedings $A M A I-07$. Menlo Park, Cia.

Channiak, 1: 2000. "A Maximum-lintropy-Inspired Patser". Procecedings ANI,P-NAACI'2000). Scittle, Wathington.

Chitrao, M. and R. Grishman, 1990). "Statistical Parsing of Messages", Proceedings IMRPA Sipeceh and language Workhop, 1990.

Collins, M. 1997. "Three generative lexicalised models for statistical parsing", procecdings EACLACL'97, Madrid, Spain.

Collins, M. 1999. Head-Driven Statistical Models for Netural Language Parsing, PhD-thesis, University of Pennsylvania, P'A.

Daclemans, W. (ed.) 1999. Memory-Based Language Processing, Joumal for Experimental and Theoretical Arificial Intelligence, 11(3).

Eisner, J. 1997. "Bilexical Grammars and a Cubic-Time Probabilistic Parser", Procedings Fifth International Workshop on Parsing Technologies, Boston, Mass.

Goodman, J. 1998. Parsing Inside-Out, Ph. D. thesis, larvard University, Mass.

Hoogweg, L. 2000. Extending DOPl with the Insertion Operation, Master's thesis, University of Amsterdam, The Netherlands.

Johnson, M. 1998. "The DOP Lstimation Method is Biased and Inconsistent", squib.
Lari, K. and S. Young 1990). "The Estimation of Stochastic Context-firee Grammats Using the Inside-Outside Algorithm", Computer Spech and I.anguage, 4, 35-56.

Magerman, D). and M. Marcus, 1991. "Pearl: A Probabilistic Chart Parser", Proceedings EACL'9l, Berlin, Gimany.

Marcus, M., B. Santorini and M. Marcinkiewice, 1993. "Building a Large Annotated Corpus of Linglish: the Pemn 'l'rechank", Compatational lingaistics 19(2).

Neumann, G. 1998. "Automatic Extraction of Stochastic Iexicalized Tree Grammars from Trecbanks", Procedings of the Ath Workstop on Tree-Adjoining (irammars and Related Frameworks, lhiladelphia, PA.

Neumann, (i. and D). Flickinger, 1999. "Learning Stochastic lexicalized free Grammars from HPSG", IJFKI Technical Report, Saarbrïicken, Gemany.

Ratnaparkhi, A. 1999. "Leaning to Parse Naltural Language with Maximum Jintropy Models", Machine Learning $34,151-176$.

Shanon, C. 1948. A Mathematical Theory of Communication. Bell System Tedhical tommal. 27, 379-423, 623656.

Sima'an, K. 1995. "An optimized algorillom for Data Oriented Parsing". in: R. Milkov and N. Nicolov (eds.), Recent Advances in Natural Language Processing 1995, volume 136 of Comrent lssues in Linguistic Theory. John Benjamins, Amsterdam.

Sima'an, K. 1996. "Computational Complexity of Probabilistic I)isambiguation by means of Tree Grammars", Procedings (o) IN(;-96, Copenhagen, Demmark.

Sima'an, K. 1999. Ieaming Efficiont Disambiguation. II. I.C Dissertation Series 1999-02, Utrecht University / University of Amsterdam, March 1999, The Netherlands.

Weischedel, R., M. Meteer, R, Schwarz, L. Ramshaw and J. Palmucei, 1993. "Coping with Ambiguity and Unknown Words through probabilistic Models", Computational Linguistics, 19(2), 359-382.

Way, A. 1999. "A Hybrid Architecture for Robust MT" using $\mathrm{L} \mathrm{FG}-\mathrm{DOOP} "$, Joumal of Experimental and Theoretical Arificial Intelligence 11 (Special lsste on Memory Based Language Processing).

Zipt, G. 1935. The Psycho-Biology of Language, Houghton Mifflin. 